\title{
Fibromuscular dysplasia presenting as spontaneous coronary artery dissection
}

\section{Background}

Coronary fibromuscular dysplasia (CFMD) is an overlooked diagnosis. Spontaneous coronary artery dissection (SCAD) may be superimposed on CFMD leading to acute coronary syndrome (ACS). ${ }^{1}$ Importantly, CFMD has been associated with non-atherosclerotic myocardial ischemia and sudden death., ${ }^{2,3}$ We report a case of spontaneous coronary artery dissection in the setting of coronary fibromuscular dysplasia.

\section{Case}

A 50years old lady with history of untreated hypertension presented with acute substernal chest pain, radiating to the center of her back following ingestion of cold water, associated with numbness in both upper extremities. Initial Electrocardiogram (ECG) showed inferior STEMI and the patient received loading doses of Aspirin and Clopidrogel. Cardiac enzymes were elevated with a Troponin-I of $26 \mathrm{ng} / \mathrm{ml}$. Emergent left heart catheterization was performed and did not reveal any obstructive coronary artery disease, yet the ostium of the right coronary was thought to have spasm and non-selective injection was performed without revealing any obvious abnormality. Hence, a Computerized Tomography (CT) aortography of the chest was ordered and aortic dissection was ruled out. A transthoracic echocardiogram revealed left ventricular ejection fraction (LVEF) of $50 \%$ with inferior wall and apico-septal hypokinesis. Due to the unexplained etiology of her presentation, the patient was transferred to a tertiary care hospital for further evaluation. Patient remained electrically and hemodynamically stable throughout her hospitalization despite the presence of intermittent chest pain episodes. This triggered a repeat left heart catheterization, which demonstrated spontaneous spiral dissection in the mid portion of the Posterior Descending Artery (PDA) (Figure 1) not previously seen by initial limited coronary angiography. Due to Concerns about the association of spontaneous dissection with Fibromuscular Dysplasia (FMD), an abdominal aortogram with bi-iliac angiogram was performed revealing right renal artery FMD (Figure 2).

Further evaluation of FMD with CT angiography of head and neck showed findings suggestive of dissection with small pseudoaneurysms involving both internal carotid arteries consistent with the diagnosed FMD. Patient was then transferred to a regular floor where she was further monitored and optimized on medical therapy. During her hospital stay, she did not have elevated blood pressure or another attack of chest pain. She was then discharged home the following day in a stable condition.

\section{Discussion}

FMD is a non-inflammatory and non-atherosclerotic vascular disease that can affect any arterial bed in the body but most commonly the renal and internal carotids. This disease predominantly affects middle-aged women but may occur at any age in both genders. ${ }^{4}$ The
Volume 9 Issue 3 - 2017

\author{
Jarrah Alfadhli,' Loulwa Alsharhan,' Claudia \\ Martinez ${ }^{2}$ \\ 'Department of Medicine, University of Miami Miller School of \\ Medicine, USA \\ ${ }^{2}$ Cardiovascular Division, University of Miami Miller School of \\ Medicine, USA
}

Correspondence: Jarrah Alfadhli, Department of Medicine, University of Miami Miller School of Medicine, Miami, Florida, USA, Email jarrah.alfadhli@jhsmiami.org

Received: June 05, 2017 | Published: July 10, 2017

United States (US) registry for FMD results for 447 patients from 9 US sites revealed that $91 \%$ of patients were females with a mean age of diagnosis of 51.9 and there was no significant difference in age at diagnosis between men and women. ${ }^{5}$ The first case of CFMD that was mentioned to be associated with SCAD was diagnosed on autopsy $1987 .{ }^{6}$ It was reported recently that $25 \%$ of women below the age of 50 presenting with myocardial infarction (MI) have SCAD. ${ }^{7}$ Most patients of FMD have "string of beads" appearance in renal, carotids, and other arterial beds on imaging but this appearance rarely occurs in coronary arteries. ${ }^{4}$ Therefore, a higher likelihood of CFMD is reached upon evaluation of other body arterial beds especially in the setting of SCAD. The etiology of FMD remains unknown, with most cases having a genetic predisposition. ${ }^{5}$ Some studies reported that FMD has autosomal dominant trait with variable penetrance but with poorly understood underlying genetics. Patients with coronary involvement of FMD usually present with dissection of an epicardial artery or major branch, which clinically leads to acute coronary syndrome (ACS) or potential sudden cardiac death. ${ }^{8}$ Initial ECG findings might reflect acute ischemia with ST deviation and sometimes with elevated cardiac biomarkers. ${ }^{3}$ SCAD is categorized as type $2 \mathrm{MI}$ as per the 2012 3rd Universal definition and FMD may be identified in other arterial beds in majority of female middle aged cases presenting acutely. ${ }^{9}$ FMD should be considered in any patient without cardiac risk factors presenting with ACS or new left ventricular dysfunction, especially in middle-aged women. Symptoms depend on the arterial bed affected with FMD and this is most often hypertension if the renal artery is affected, headache, or pulsatile tinnitus if cervical vessel involvement. $^{8}$

Currently, only catheter-based angiography is validated for diagnosing coronary artery involvement as no studies documented the utility of other noninvasive imaging modalities for diagnosis CFMD. ${ }^{8}$ Intravascular Ultrasound (IVUS) and Optical Coherence Tomography (OCT) are excellent adjunctive imaging modalities 
for use in combination with angiography but they are not used that often unless there was difficulty in diagnosing CFMD. Physicians should have higher index of suspicion in patients who do not have cardiovascular risk factors and in patients that have other circulatory findings suggestive of FMD. Although not All SCAD patients have the diagnosis of CFMD, excluding FMD with routine imaging of renal, iliac, and head CTA are required as involvement have longterm consequences.

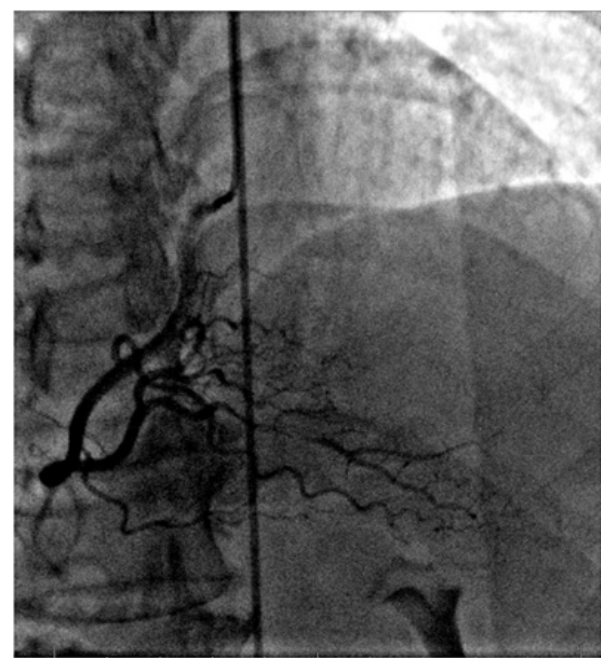

Figure I Still frame image of right coronary artery terminating into a tortuous posterior descending artery where there is a distal dissection (blue arrow).

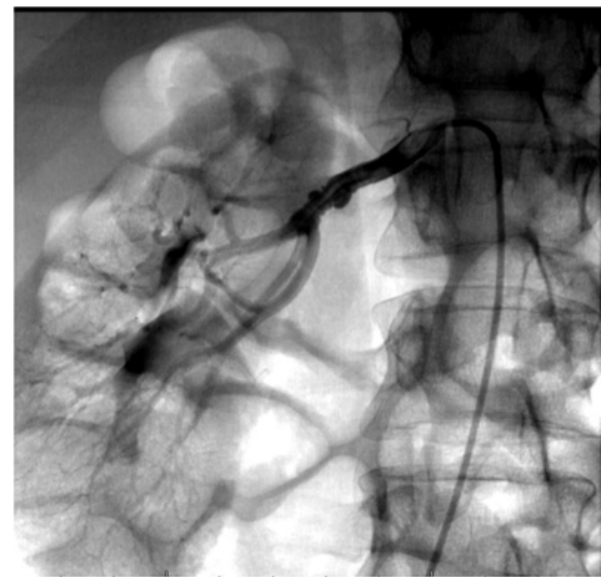

Figure 2 Still frame conventional angiography showing typical beaded appearance of the renal artery (blue arrow). Differential considerations include FMD, atheromatous disease causing aneurysmal dilatation and stenosis of the renal artery.

Treatment of CFMD is challenging, as there are limited objective and scientifically based guidance due to absence of prospective trials or large observational case series. Therefore, treatment based on case reports and limited case series. ${ }^{8}$ In stable patients with no MI, conservative approach is preferred, as SCAD will typically heal spontaneously. Stenting is challenging in most of the cases and unless ultrasound guided, might make the dissection flap propagate beyond the stented segment and/or intramural hematoma may arise..$^{10,11}$ However, in unstable patients with compromised distal coronary flow, interventional approach may be required and in these cases ultrasound guidance is required in order to guide position of stent in true lumen. ${ }^{12}$ Coronary artery bypass graft $(\mathrm{CABG})$ should be reserved for those patients that have multiple vessels involvement with good distal target vessels. ${ }^{11}$ The role of antithrombotic in SCAD is unknown but dual antiplatelet therapy with aspirin and clopidogrel for 1 year is recommended followed by aspirin alone indefinitely if there are no bleeding complications. ${ }^{13,14}$ Beta-blocker may be considered given the rationale of reducing coronary shear forces by reducing the blood pressure and heart rate..$^{13}$ Hypertension, whether attributed to renal artery FMD or not, should be treated to goal. ${ }^{5}$ Statins were not proven yet to be beneficial in patients with FMD and/or dissection as per published guidelines thus their use should be with the goal of treating dyslipidemia if present. ${ }^{5,15}$ Avoidance of strenuous physical activity and heavy weights lifting is generally advised given the rare association with SCAD. ${ }^{8}$

In this case we presented an uptodate review of the available literature on CFMD and its association with ACS. Further prospective trials to delineate the potential causal link between SCAD and FMD, diagnosing approach, and management are required.

\section{Acknowledgements}

None.

\section{Conflicts of interest}

Author declares there are no conflicts of interest.

\section{Funding}

None.

\section{References}

1. Saw J, Rohan P, Anthony F, et al. Spontaneous Coronary Artery Dissection in Patients With Fibromuscular Dysplasia. A Case Series .2012;5(1):134-137.

2. Michaud K, Romain N, Brandt-Casadevall C, et al. Sudden death related to small coronary artery disease. Am J Forensic Med Pathol . 2001;22(3):225-227.

3. Pate GE, R Lowe, CE Buller. Fibromuscular dysplasia of the coronary and renal arteries? Catheterization and Cardiovascular Interventions. 2005;64(2):138-145.

4. Slovut DP, JW Olin. Fibromuscular dysplasia. $N$ Engl $\mathrm{J}$ Med. 2004;350(18):1862-1871.

5. Olin JW, Froehlich J, Gu X, et al. The United States Registry for Fibromuscular Dysplasia: results in the first 447 patients. Circulation . 2012;125(25):3182-3190.

6. Lie JT, KK Berg. Isolated fibromuscular dysplasia of the coronary arteries with spontaneous dissection and myocardial infarction. Hum Pathol. 1987;18(6):654-656.

7. Saw J, Aymong E, Mancini GB, et al. Nonatherosclerotic coronary artery disease in young women. Can J Cardiol. 2014;30(7):814-819.

8. Michelis KC, Olin JW, Kadian-Dodov D, et al. Coronary artery manifestations of fibromuscular dysplasia. $\mathrm{J} \mathrm{Am}$ Coll Cardiol.2014;64(10):1033-1046.

9. Thygesen K, Joseph S Alpert, Allan S Jaffe, et al. Third Universal Definition of Myocardial Infarction. Journal of the American College of Cardiology. 2012;60(16):1581-1598.

10. Saw J, Ricci D, Starovoytov A, et al. Spontaneous coronary artery dissection: prevalence of predisposing conditions including fibromuscular dysplasia in a tertiary center cohort. JACC Cardiovasc Interv. 2013;6(1):444-452.

11. Tweet MS, Hayes SN, Pitta SR, et al. Clinical features, management, and prognosis of spontaneous coronary artery dissection. Circulation. 2012;126(5):579-588. 
12. Poulter R, D Ricci, J Saw. Perforation during stenting of a coronary artery with morphologic changes of fibromuscular dysplasia: an unrecognized risk with percutaneous intervention. Can J Cardiol. 2013;29(4):519. e1-519.e3

13. Saw J . Spontaneous Coronary Artery Dissection. Can J Cardiol.2013;29(9):1027-1033.

14. Choi JW, CJ Davidson. Spontaneous multivessel coronary artery dissection in a long-distance runner successfully treated with oral antiplatelet therapy: A case report and review of the literature. Journal of Invasive Cardiology. 2002;14(11):675-678.
15. $2011 \mathrm{ACCF} / \mathrm{AHA}$ focused update of the guidelines for the management of patients with unstable angina/non-st-elevation myocardial infarction (updating the 2007 guideline). Journal of the American College of Cardiology. 2011;57(19):1920-1959. 